\title{
Finite elements apparatus in thin-walled rods dynamics problems
}

\author{
Vladimir Rybakov ${ }^{1 *}$, Stanislav Dyakov ${ }^{1}$, Daniil Sovetnikov ${ }^{1}$, Artur Azarov $^{1}$, and Sergey \\ Ivanov $^{1}$ \\ ${ }^{1}$ Peter the Great St. Petersburg Polytechnic University, Politekhnicheskaya, 29, Saint-Petersburg, \\ 195251, Russia
}

\begin{abstract}
The calculation of thin-walled rods is extremely relevant problem of structural mechanics and not only from the scientific standpoint, but also due to the widespread use of so-called lightweight thin-walled steel structures for construction engineering sector. Regardless of a sufficiently large number of studies connected with the statics of thin-walled rods, the dynamics of such systems have not been thoroughly studied yet. Based on one of the forward-looking theories of calculation i.e. the semi-shear theory by Slicker, the paper provides a technique for solving the dynamics problems of thin-walled rods. The stiffness and mass matrices of the finite element system are obtained for linear approximation of the form functions, and the natural vibration frequencies of the rods are calculated. The obtained solution is accomplished by the extrapolation method of estimating the accuracy of numerical methods for solving mathematical problems.
\end{abstract}

\section{Introduction}

The problem of thin-walled beam calculation is particularly urgent at the present time not only because of a scientific interest, but also considering a very wide use of so-called light steel thin-walled constructions in modern building practice; such constructions have very small thickness (below $4 \mathrm{~mm}$ ) and that is why traditional beam is not suitable for a correct description of stress-deformation conditions and behavior under different loads.

Let us point out the most common Russian theories of thin-walled beam calculation:

1. Non-shear Vlasov theory for open section thin-walled beams calculation which main assumption is to neglect shear deformation in beam middle surface.

2. Umanskiy theory is suitable for closed cross section thin-walled beams. This theory differs from the previous one by the assumption that longitudinal displacement of the beam due to transfer are proportional (by some coefficient) to displacements due to free torsion. This assumption allows us to get the system of final equations describing twisting of a thinwalled beam with closed cross-section.

3. According to Slivker semi-shear theory tangential stresses in beam cross-section are presented in a form of superposition of bending stresses (due to transverse forces) and torsion stresses (due to torsion moment $M_{k}$ and moment of restricted torsion (sectorial torsion

\footnotetext{
*Corresponding author: fishermanoff@mail.com
} 
moment) $M_{\omega}$ ). Thus, Slivker suggests us to neglect the first part of tangential stresses as those stresses do not make much difference in total stresses. After all, the scientist was able to develop the theory that allows calculating thin-walled beams of any kind of cross-section.

In general, many problems of thin-walled beams statics are described and solved in different scientific papers.

In paper [5] stress-deformation analysis of thin-walled structural elements is carried out for open and closed cross-section. The degree of influence of transfer on general stressdeformation condition of thin-walled beam was analyzed.

Results of derivation and testing of thin-walled beam finite elements (FE) for open crosssection under torsion influence considering shear deformation were published in [6].

Scientific papers $[7,8]$ by Belyh outlined the distinctive features of the work of elements of steel structures made of cold-formed galvanized profiles. Calculations for strength and stability, considering the reduced cross-section, are made using Eurocode-3.

Considering [9], the possibility of using local plastic mechanisms for characterizing the time resistance of short thin-walled cold-rolled steel elements subjected to eccentric compression with respect to a minor axis of symmetry of the cross section was investigated.

As for paper [10], a new stress-deformation model of elastic and inelastic local bending of thin-walled steel elements, based on the energy method, allowed to calculate the maximum strength and local bending of thin-walled beams under axial compressive force.

A simple analytical solution for the stresses and displacements of short thin-walled columns of open bisymmetric sections subjected to eccentric axial loads was proposed in [11]. Analytical solutions are obtained for closed profile for both stresses and displacements. The results of the paper are compared with numerical tests performed using the FE shell system.

Numerical analysis allowed to obtain the values of critical compressed loads for thin walled profiles with different parameters under the influence of various loads were described in articles [12-14].

Influence of variety of geometric characteristic such as stiffness on strength of joints made of thin-walled profiles was described in studies [15-16].

The problems of evaluation of bearing capacity of thin-walled systems used in specific constructive solutions were discussed in detail in the articles [17-18].

Researches on thin-walled beams under dynamic influence have been carried out less often:

One of the first works devoted to the oscillations of thin-walled beams was a thesis by Wai Keung [19]. The author considered torsional and flexural vibrations of thin-walled beams of asymmetric cross section with allowance for the shear deformation, what influences on accurate calculation of short length beams.

Three theories of torsional oscillations of homogeneous thin-walled beams in the energy formulation were considered in [20]. Dispersion dependencies and phase velocities of torsional waves were analyzed for each of the theories.

Geometrically nonlinear problems of oscillations of thin-walled beams were considered in [21-22]. As a result, the author obtained a stiffness matrix for a two-node FE with seven degrees of freedom at each node.

The fact that in the modern construction industry the main method of calculating constructions (including thin-walled elements) is the FE method cannot be a discovery for anybody. Despite its relative simplicity and utility, it is distinguished by some approximation of the results. Logically, the accuracy of the results depends on the geometry and the number of FE of a given type. Therefore, it is extremely important to correctly break down the model into FE, for which purpose the most optimal FE step should be determined.

The studies of the stability of structures using the FE method were presented in the works [23-24]. 
Practical methods of solving problems of constrained torsion without the use of complex mathematical apparatus were described in the article [25]. The formula allowed to determine bimoment using the known values of the bending moment and the eccentricity of applied load was given.

The variational problem of structural mechanics was considered in [26].

The surrogate models and the basic techniques of their construction to calculate initial rotational stiffness of welded RHS joints made of high strength steel (HSS) were represented in [27-28].

The problems of the convergence of the static problems solution were described in [29].

However, the problem of the convergence of the dynamic problem solution has never been studied.

For this reason, a special interest for the authors of this paper is drawn to the problem of estimating the accuracy of the finite element method for dynamic problems, based on the analysis of the model with several steps of the finite element decomposition, connected with each other by certain mathematical dependencies.

The purpose of this study is to determine the method of implementation of finite elements apparatus for solving dynamic problems using Slivker semi-shear theory and Schwarzmann dual convergence method

The stiffness matrix for finite element with seven degrees of freedom in each node is derived for the corresponding mass matrix, and the accuracy of the solution is estimated by the Schwarzmann method using calculated frequencies.

\section{Methods}

In this work the problem of finding the natural frequencies of the thin-wall profile was solved.

The boundary conditions will take as a beam with tight fitting from one end (Fig. 1).

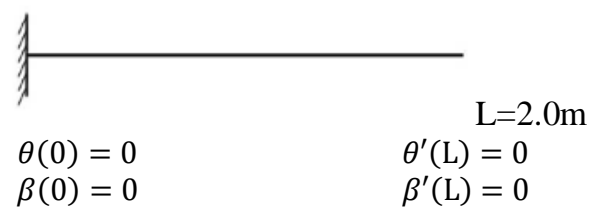

Fig. 1. Design scheme

The calculations were produced considering shear strain based on semi-shearing Theory of Slivker, which can detect two dispersion branches of rod vibrations: acoustic and optical. Obviously, this is an advantage of this theory over shear-free Vlasov Theory.

Cross section of the beam consists of two c-shaped profiles called PN150-50-1.5 (height $-150 \mathrm{~mm}$, width $-50 \mathrm{~mm}$, thickness $-1.5 \mathrm{~mm}$ ). Profiles are connected together by welding which allows both parts to work jointly as a whole rod. Geometric and material properties for fabricated cross section (Fig. 2) are given in table 1.

The solution was found in an approximate form using the energy approach. In contrast to the analytical (exact) solution, this method does not complicate with excessive complexity of calculations and does not also have many restrictions: constant stiffness along the length of the rod, limited type of loads, etc. 
Table 1. Geometric and material properties

\begin{tabular}{|l|c|c|c|}
\hline \multicolumn{1}{|c|}{ Parameter } & Value & Unit \\
\hline Cross area & $A$ & 8.428 & $\mathrm{~cm}^{2}$ \\
\hline Moment of inertia at free torsion & $I_{d}$ & $3.9206 \cdot 10^{-6}$ & $\mathrm{~cm}^{2}$ \\
\hline Sectorial moment of inertia & $I_{\omega}$ & 1378.266 & $\mathrm{~cm}^{6}$ \\
\hline The moment of inertia about Central axis Y1 & $I_{y}$ & 289.239 & $\mathrm{~cm}^{4}$ \\
\hline Moment of inertia about the Central axis Z1 & $I_{z}$ & 50.123 & $\mathrm{~cm}^{4}$ \\
\hline Polar moment of inertia & $I_{r}$ & 339.362 & $\mathrm{~cm}^{4}$ \\
\hline Steel density & $\rho$ & 7858 & $\mathrm{~kg} / \mathrm{m}^{3}$ \\
\hline Shape factor & $\psi$ & 1.00014 & \\
\hline Young's modulus & $E$ & $2 \cdot 10^{11}$ & $\mathrm{~Pa}$ \\
\hline Poisson ratio & $v$ & 0.3 & \\
\hline
\end{tabular}

\subsection{Construction of Mass matrix}

Expression of kinetic energy for a straight-line thin-wall rod of length 1 operating on torsion taking with consideration of shear strain is given by [4]:

$$
T=\frac{1}{2} \int_{V} \rho\left(\frac{d \underline{U}}{d t}\right)^{2} d V
$$

where $\rho$ - volume density of a material;

$\mathrm{U}$ - total displacement vector;

$\mathrm{V}$ - volume of the rod.

In further calculations we will record the derivative $\frac{d F}{d t}$ as $\dot{F}$ for convenience.

The same expression after a series of transformations:

$$
\begin{gathered}
T=\frac{1}{2} \int_{L}\left[\rho_{L} \dot{U}_{o x}^{2}+\rho_{L} \dot{U}_{o y}^{2}+\rho_{L} \dot{U}_{o z}^{2}+\rho_{L} \dot{\theta}^{2}\left(z_{p}^{2}+y_{p}^{2}\right)+\rho \dot{\theta}^{2} I_{r}+\rho \dot{\beta}^{2} I_{\omega}+2 \rho_{L} z_{p} \dot{U}_{o y} \dot{\theta}\right. \\
\left.-2 \rho_{L} y_{p} \dot{U}_{o z} \dot{\theta}\right] d x
\end{gathered}
$$

where $\theta(x)$ - the angle of rotation of the section as a rigid body; $\beta(x)$-deplanation measure function; $\rho_{L}-$ linear density; $\mathrm{y}_{\mathrm{p}}, \mathrm{z}_{\mathrm{p}}$ - distance from the center of section bend to the central axis of inertia; $U_{o x}, U_{o x}, U_{o x}$ - linear movements along the axes.

The expression (2) was obtained in view of the fact, that $y$ and z-axes are the main central axes of the section, the main pole was chosen as the pole, and the main initial point of the section was chosen as the starting point.

Present functions of movement in the form

$$
F(x, t)=F_{0}(x) \cos (\omega t)
$$

Where (2) is converted to the form:

$$
\begin{gathered}
T=\omega^{2} \cos (\omega t) \frac{1}{2} \int_{L}\left[\rho_{L} U_{o x}^{2}+\rho_{L} U_{o y}^{2}+\rho_{L} U_{o z}^{2}+\rho_{L} \theta^{2}\left(z_{p}^{2}+y_{p}^{2}\right)+\rho \theta^{2} I_{r}+\rho \beta^{2} I_{\omega}\right. \\
\left.+2 \rho_{L} z_{p} U_{o y} \theta-2 \rho_{L} y_{p} U_{o z} \theta\right] d x
\end{gathered}
$$

Authors approximated functions of displacements in accordance with Ritz Method by using shape functions mentioned in table 2.

Table 2. Approximation of displacement functions

\begin{tabular}{|c|c|c|c|}
\hline Shape function type & Linear & \multicolumn{2}{|c|}{ Cubical } \\
\hline Approximated displacements & $\theta, \beta, U_{o x}$ & \multicolumn{2}{|c|}{$U_{o y}, U_{o z}$} \\
\hline \multirow{3}{*}{ Expressions for functions } & $N_{1}=1-\frac{x}{l}$ & $N_{3}=\frac{2}{l^{3}} x^{3}-\frac{3}{l^{2}} x^{2}+1$ & $N_{5}=-\frac{2}{l^{2}} x^{3}+\frac{3}{l} x^{2}$ \\
\cline { 2 - 4 } & $N_{2}=\frac{x}{l}$ & $N_{4}=\frac{1}{l^{2}} x^{3}-\frac{2}{l} x^{2}+\mathrm{x}$ & $N_{6}=\frac{1}{l^{2}} x^{3}-\frac{1}{l} x^{2}$ \\
\hline
\end{tabular}


Movements included in the functional (4) will be written in the form:

where $a_{i}$ - displacement in the $i$-th node.

$$
F(x)=\sum a_{i} N_{i}
$$

Each displacement in the form of (5) need to be substituted in the functional (2), which after calculating of certain integrals will turn into an ordinary function of variables $a_{i}$.

$$
\frac{\partial T}{\partial a_{i}}=0
$$

Then having calculated corresponding partial derivatives (5), we obtained a matrix of coefficients - the mass matrix M (Fig.3).

$$
\begin{aligned}
& m_{1,1}=m_{8,8}=\frac{l \rho_{L}}{3} ; \quad m_{1,8}=\frac{l \rho_{L}}{6} ; \quad m_{2,2}=m_{9,9}=m_{3,3}=m_{10,10}=\frac{13 l \rho_{L}}{35} ; m_{2,4}=\frac{7 l z_{p} \rho_{L}}{20} ; m_{2,6}=\frac{11 l^{2} \rho_{L}}{210} ; \quad m_{2,9}=\frac{9 l \rho_{L}}{70} ; \\
& m_{2,11}=m_{4,9}=\frac{3 l z_{p} \rho_{l}}{20} ; \quad m_{2,13}=m_{5,10}=\frac{-13 l^{2} \rho_{L}}{420} ; \quad m_{3,4}=m_{10,11}=-\frac{7 l y_{p} \rho_{L}}{20} ; \quad m_{3,5}=m_{10,12}=-\frac{11 l^{2} \rho_{L}}{210} ; \\
& m_{3,10}=m_{9,11}=\frac{9 l \rho_{L}}{70} ; \quad m_{4,4}=\frac{l_{r} l \rho}{3}+\frac{l y_{p}^{2} \rho_{L}}{3}+\frac{l z_{p}^{2} \rho_{L}}{3} ; m_{4,5}=\frac{l^{2} y_{p} \rho_{L}}{20} ; \quad m_{4,6}=\frac{l^{2} z_{p} \rho_{L}}{20} ; \quad m_{4,10}=-\frac{3 l y_{p} \rho_{L}}{20} ; \\
& m_{4,11}=\frac{l_{r} l \rho}{6}+\frac{l y_{p}^{2} \rho_{L}}{6}+\frac{l z_{p}^{2} \rho_{L}}{6} ; m_{4,12}=\frac{l^{2} y_{p} \rho_{L}}{30} ; \quad m_{4,13}=-\frac{l^{2} z_{p} \rho_{L}}{30} ; m_{5,5}=m_{6,6}=m_{12,12}=m_{13,13}=\frac{l^{3} \rho_{L}}{105} ; \\
& m_{5,11}=\frac{l^{2} y_{p} \rho_{L}}{30} ; m_{5,12}=\frac{l^{3} \rho_{L}}{140} ; \quad m_{6,9}=\frac{13 l^{2} \rho_{L}}{420} ; \quad m_{6,11}=\frac{l^{2} z_{p} \rho_{L}}{30} ; \quad m_{6,13}=-\frac{l^{3} \rho_{L}}{140} ; \quad m_{7,7}=m_{14,14}=\frac{l_{\omega} l \rho}{3} ; \\
& m_{7,14}=\frac{l_{\omega} l \rho}{6} ; \quad m_{11,11}=\frac{l_{r} l \rho}{3}+\frac{l y_{p}^{2} \rho_{L}}{6}+\frac{l z_{p}^{2} \rho_{L}}{6} ; m_{11,12}=\frac{l^{2} y_{p} \rho_{L}}{20} ; m_{11,13}=\frac{l^{2} z_{p} \rho_{L}}{20} .
\end{aligned}
$$

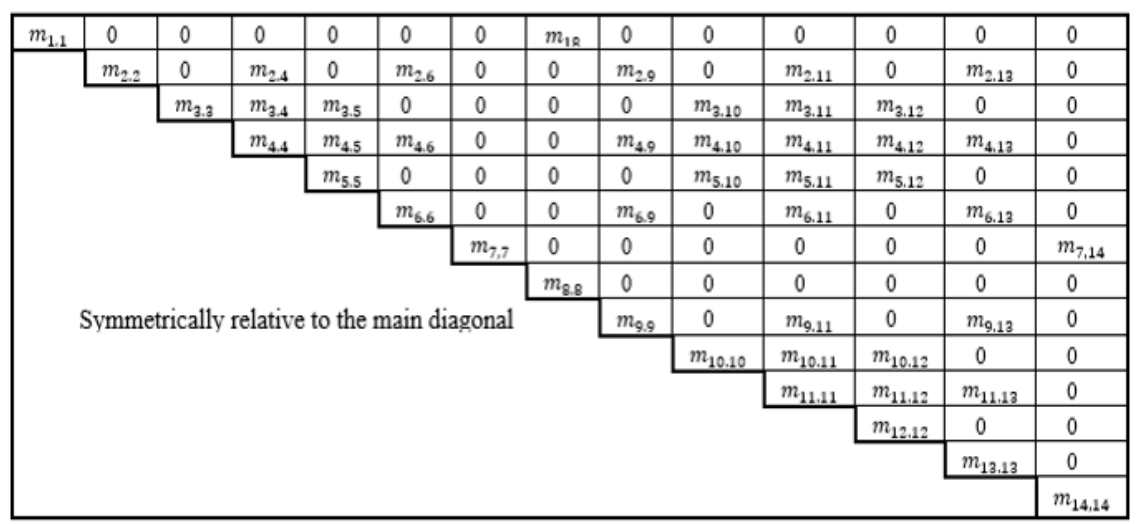

Fig. 3. Mass matrix of thin-walled rods system with $7 \mathrm{deg}$. of freedom in nodes

\subsection{Calculation of oscillation frequencies}

The same approximating functions as in the construction of the mass matrix (Table 2) were used to construct the stiffness matrix $\mathrm{K}$. The construction of standard view stiffness matrix for rod with 7 degrees of freedom in each node was parsed in the article [5].

The equation of natural oscillations is written in the form:

$$
\left(K^{S U M}-\varpi^{2} M^{S U M}\right) U=0,
$$

where: $K^{S U M}$ - general stiffness matrix of the system; $M^{S U M}$ - general mass matrix of the system; $U$ - nodal displacement vector; $\varpi-$ natural frequency vector.

For existence of nontrivial solutions, the condition must be satisfied:

$$
\left|K^{S U M}-\varpi^{2} M^{S U M}\right|=0
$$

If $n$ is the order of matrices, $K^{S U M}$ and $M^{S U M}$ then the determinant will be a polynomial of the $n$-th order which will have $n$ roots: $\varpi_{\mathrm{i}}$. These roots are squares of eigenfrequencies.

The results of frequency counting are given in the Table 3 
Table 3. Thin-walled profile oscillation frequencies

\begin{tabular}{|c|c|c|c|c|c|c|c|c|}
\hline \multicolumn{2}{|c|}{ No. of frequency } & $2 \mathrm{FE}$ & $4 \mathrm{FE}$ & $8 \mathrm{FE}$ & $16 \mathrm{FE}$ & $32 \mathrm{FE}$ & $64 \mathrm{FE}$ & $128 \mathrm{FE}$ \\
\hline \multirow{7}{*}{ 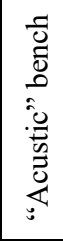 } & 1 & 713.86 & 373.82 & 205.80 & 133.29 & 107.72 & 100.30 & 98.36 \\
\hline & 2 & 4967.64 & 2469.52 & 1287.93 & 807.94 & 639.31 & 590.07 & 577.13 \\
\hline & 3 & & 7597.86 & 3706.27 & 2253.09 & 1763.96 & 1622.67 & 1585.64 \\
\hline & 4 & & 15311.15 & 7591.38 & 4423.68 & 3419.24 & 3134.58 & 3060.33 \\
\hline & 5 & & & 13211.61 & 7346.38 & 5586.77 & 5099.72 & 4973.50 \\
\hline & 6 & & & 20625.42 & 11047.02 & 8239.00 & 7481.60 & 7286.79 \\
\hline & 7 & & & 29139.97 & 15555.71 & 11348.02 & 10242.18 & 9960.03 \\
\hline \multirow{7}{*}{$\begin{array}{l}\frac{\tilde{0}}{0} \\
0 \\
0 \\
\overline{0} \\
0 \\
0 \\
0 \\
0\end{array}$} & 1 & 142180.10 & 142218.17 & 142255.27 & 142282.54 & 142049.81 & 142287.55 & 142245.64 \\
\hline & 2 & 142785.78 & 142915.90 & 143014.99 & 143103.77 & 142392.84 & 142717.26 & 142863.19 \\
\hline & 3 & & 144287.35 & 144350.51 & 144476.59 & 143213.48 & 143547.86 & 143304.69 \\
\hline & 4 & & 146000.12 & 146349.18 & 146407.70 & 144719.89 & 144679.49 & 144827.72 \\
\hline & 5 & & & 149077.64 & 148907.14 & 146491.56 & 146746.29 & 146363.67 \\
\hline & 6 & & & 152446.79 & 151988.16 & 147851.65 & 148361.82 & 148248.72 \\
\hline & 7 & & & 155948.70 & 155665.16 & 149278.99 & 150333.64 & 149326.90 \\
\hline
\end{tabular}

\section{Results and Discussion}

\subsection{Comparison of the frequency of oscillations}

The eigenfrequencies calculated using method described above were compared with the results obtained in work [20].

When comparing, it was taken into account, that author has chosen nonthin-walled axisymmetric profile - I-beam with dimensions of $43 \times 40 \mathrm{~cm}$ with a wall thickness of $3 \mathrm{~cm}$.

The results obtained in works [20] are presented in table 4.

Table 4. Profile with thick walls oscillation frequencies

\begin{tabular}{|c|c|c|c|c|c|c|}
\hline & No. of frequency & $2 \mathrm{FE}$ & $4 \mathrm{FE}$ & $8 \mathrm{FE}$ & $16 \mathrm{FE}$ & $32 \mathrm{FE}$ \\
\hline \multirow{7}{*}{ 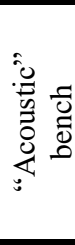 } & 1 & 604.28 & 264.44 & 132.74 & 75.63 & 53.057 \\
\hline & 2 & & 1211.5 & 535.9 & 279.03 & 173.83 \\
\hline & 3 & & 3188.5 & 1273.9 & 625.07 & 373.58 \\
\hline & 4 & & & 2446.4 & 1125.7 & 654.27 \\
\hline & 5 & & & 4162.9 & 1797.3 & 1017.5 \\
\hline & 6 & & & 6398.8 & 2661.2 & 1465.1 \\
\hline & 7 & & & 8633.1 & 3742.3 & 1999.4 \\
\hline \multirow{7}{*}{ 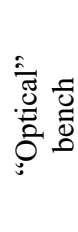 } & 1 & 71005 & 71005 & 71005 & 71005 & 71005 \\
\hline & 2 & 71034 & 71032 & 71031 & 71031 & 71031 \\
\hline & 3 & 71093 & 71124 & 71115 & 71112 & 71111 \\
\hline & 4 & & 71275 & 71261 & 71248 & 71245 \\
\hline & 5 & & 71358 & 71479 & 71443 & 71432 \\
\hline & 6 & & & 71765 & 71699 & 71647 \\
\hline & 7 & & & 72077 & 72022 & 71970 \\
\hline
\end{tabular}

However, comparing the results, it is easily notice the similarity of the eigenfrequency function behavior, as well as the clear division of frequencies into two branches: acoustic and optical.

\subsection{Convergence estimate of finite elements}

The Schwarzman method chosen by authors of the study to estimate the error of the obtained natural frequencies is favorable because the method proposed by the scientist [29, 30], unlike the methods of Richardson or Aitken, does not contain indefinite constants.

The theorem formulated by Schwarzman in [29] resembles the following: 
"If the sequence $\mathrm{D}_{\mathrm{i}}=\frac{\left(\mathrm{z}_{\mathrm{i}+1}-\mathrm{z}_{\mathrm{i}}\right)}{\left(\mathrm{z}_{\mathrm{i}+2}-\mathrm{z}_{\mathrm{i}+1}\right)} \quad\left(z_{i}-\right.$ the value of the approximate solution at some point of the problem's definition) monotonically converges to $2^{k}$, then the exact solution $z_{\mathrm{T}}$ belongs to the segment determined by the values $R_{i}=z_{i+2}+\frac{\left(z_{i+2}-z_{i+1}\right)}{2^{k}-1}$ and $A_{i}=z_{i+2}+$ $\frac{\left(z_{i+2}-z_{i+1}\right)}{D_{i}-1}$ “

i. e. the following is true:

$$
\min \left(A_{i}, R_{i}\right) \leq z_{T} \leq \max \left(A_{i}, R_{i}\right)
$$

In addition, if a final approximate solution is represented in the form:

$$
S_{i}=\frac{\left(A_{i}+R_{i}\right)}{2}
$$

the following error estimation will be sufficient:

$$
e=\left|z_{T}-S_{i}\right| \leq \frac{\left|A_{i}-R_{i}\right|}{2}
$$

Estimation of the error of the obtained natural frequencies is presented in table 5.

Table 5. Estimation of the error of the obtained natural frequencies

\begin{tabular}{|c|c|c|c|c|c|c|}
\hline $\mathrm{N}_{\mathrm{FE}}$ & $\varpi$ & $\mathrm{D}$ & $\mathrm{R}$ & $\mathrm{A}$ & $\mathrm{S}$ & $\mathrm{e}$ \\
\hline \multicolumn{7}{|c|}{$1^{\text {st }}$ frequency of «Acoustic» bench } \\
\hline 2 & 713.86 & & & & & \\
\hline 4 & 373.82 & & & & & \\
\hline 8 & 205.80 & 2.0238 & 149.7934 & 41.687 & 95.740 & 54.053 \\
\hline 16 & 133.29 & 2.3172 & 109.1200 & 78.241 & 93.681 & 15.440 \\
\hline 32 & 107.72 & 2.8357 & 99.1967 & 93.791 & 96.450 & 2.703 \\
\hline 64 & 100.30 & 3.4461 & 97.8267 & 97.267 & 97.547 & 0.280 \\
\hline 128 & 98.36 & 3.8271 & 97.7150 & 97.675 & 97.695 & 0.0198 \\
\hline
\end{tabular}

Since the sequence D converges monotonically to 4 , the absolute error of the final approximate value of the $1^{\text {st }}$ frequency of «acoustic» bench and the exact solution does not exceed e value.

\section{Conclusion}

1. The resulting mass matrix for the finite element with 7 degrees of freedom in the node considering semi-shear deformation theory of V. I. Slivker.

2. The advantage of the constructed FE is the possibility of finding the natural frequencies of oscillations of two ranges: "acoustic" (50 - $2000 \mathrm{rad} / \mathrm{s}$ ) and "optical" (from $71000 \mathrm{rad} /$ s) branches

3. The extrapolation method of B.S. Schwarzman shows the convergence of the natural frequencies of the "acoustic" branch. The recommended minimum number of FE at which the error of calculation of all eigenfrequencies does not exceed 3\% is 32 elements.

4. The possible improvement of convergence of "optical" branch is using of higher-order approximation of displacement functions.

\section{References}

1. V. Vlasov, Thin-walled elastic rods (Fizmatgiz, Moscow, 1959).

2. A. Umanskiy, Torsion and Flexure of thin-walled aircraft structures (Oboronizdat, Moscow, 1939).

3. A. Umanskiy, Calculation of thin-walled curved beams (Fizmatgiz, Moscow, 1944). 
4. V. Slivker, Structural mechanics. A variational framework. Textbook (ASV, Moscow, 2005).

5. V. Lalin, V. Rybakov, A. Sergey, Appl. Mech. and Mat. 578-579, 858-863 (2014).

6. V. Lalin, Transp. Constr. 2, 130-140. (2011).

7. G. Bely, Bull. of Civ. Eng. 4 (45), 32-37 (2014).

8. G. Bely, Bull. of Civil Eng. 3(35), 99-103. (2012).

9. H.-T. Thai, Br. Uy, Kh. Mahbub, J. of Constr. Steel Res. 111, 57-69 (2015).

10. R. Pavazza, F. Vlak, M. Vukasović, Proc. Eng. 161, 349-355 (2016).

11. L.P. Huang, Dynamics of thin-walled beams. (WCE, Carolina, 1963).

12. A. Sinelnikov, Mag. of Civ. Eng. 5(57), 74-85. (2015).

13. T. Nazmeeva., N. Vatin, Mag. of Civ. Eng. 2(62), 92-101. (2016).

14. N. Vatin, T. Nazmeeva, R. Guslinscky, Adv. Mat. Res. 941-944, 1871-1875. (2014).

15. M. Garifullin, N. Vatin, T. Jokinen, M, Heinisuo, ESaT 2016, 81-86 (2017).

16. I.V. Atavin, B.E. Melnikov, A.S. Semenov, N.V. Chernysheva, E.L. Yakovleva, Mag. of Civ. Eng. 4(80), 48-61. (2018).

17. V. Rybakov, A. Al, A. Panteleev, K. Fedotova, A. Smirnov, Mag. of Civ. Eng. 8(76), 2839. (2017).

18. V. Roy, O. Izvekova, I. Togo, MATEC Web of Conferences. 86, 142-150 (2016).

19. T. W. Keung, Dynamics of thin-walled beams of open section. (MT, California, 1964).

20. S. Dyakov, V. Lalin, Sc. of Sc. 5(18), 18-24 (2013).

21. K. Hsiao, Y. Wen, R. Chen, Proceeding of the world congress on Engineering. 2, 124139 (2009).

22. K. M. Hsiao, J. Y. Lin, W. Y. Lin, Comput. Methods Appl. Mech. Eng. 169, 1-18 (1999).

23. A.Kibkalo, M. Lebedeva, M. Volkov, MATEC Web of Conferences. 53, 256-268 (2016). 24. O.A. Tusnina, Mag. of Civ. Eng. 77(1), 68-89. (2018).

25. A. Pavlenko, V. Rybakov, A. Pikht, E. Mikhailov, Mag. of Civ. Eng. 7(67), 55-69. (2016).

26. V.Lalin, E. Zdanchuk, D. Kushova, L. Rozin, Mag. of Civ. Eng. 4(56), 54-165. (2015).

27. M. Garifullin, M. Bronzova, M. Heinisuo, K. Mela, S. Pajunen, Mag. of Civ. Eng. 4(80), 81-94 (2018).

28. M. Garifullin, A. Barabash, E. Naumova, O. Zhuvak, T. Jokinen, M. Heinisuo, Mag. of Civ. Eng. 3(63), 53-176 (2016).

29. B. Shvartsman, Constr. Mech. and calc. of str. 3, 157-162 (1992). 